\title{
ON ONE-FACTORIZATIONS OF COMPLETE GRAPHS
}

Dedicated to the memory ot Hanna Neumann

\author{
W. D. WALLIS
}

(Received 21 March 1972)

Communicated by G. Szekeres

\section{Introduction}

We use standard graph notation and definitions, as in [1]: in particular $K_{n}$ is the complete graph on $n$ vertices and $K_{n, n}$ is the regular complete bigraph of order $2 n$.

Given a graph $G$, a factor of $G$ is a spanning subgraph of $G$ and a factorization is a sequence of edge-disjoint factors whose union is $G$. A one-factor is a factor which is a regular graph of degree 1; a one-factorization is a factorization whose factors are all one-factors. It is well-known that $K_{2 n}$ and $K_{n, n}$ always have onefactorizations. If $K_{2 n}$ has vertex-set $\{1,2, \cdots, 2 n\}$ then $\left[1\right.$, p. 85] $\mathscr{G}_{2 n}=\left\{G_{1}, G_{2}, \cdots\right.$, $\left.G_{2 n-1}\right\}$ is a one-factorization where

$$
G_{i}=\{(2 n, i)\} \cup\{(i-j, i+j): j=1,2, \cdots, n-1\},
$$

$i-j$ and $i+j$ being taken as integers modulo $2 n-1$ in the range $\{1,2, \cdots, 2 n-1\}$. If the vertices of $K_{n, n}$ are written as $1_{1}, 2_{1}, \cdots, n_{1}, 1_{2}, 2_{2}, \cdots, n_{2}$ where the induced subgraph of $1_{\alpha}, 2_{\alpha}, \cdots, n_{\alpha}$ is null then $\mathscr{X}_{n}=\left\{X_{1}, X_{2}, \cdots, X_{n}\right\}$ is a one-factorization if

$$
X_{i}=\left\{\left(j_{1},(j-i+1)_{2}\right): j=1,2, \cdots, n\right\},
$$

$j-i+1$ being taken as integers modulo $n$ in the range $\{1,2, \cdots, n\}$.

Two factorizations $\mathscr{F}$ and $\mathscr{F}^{\prime}$ of $G$ are isomorphic if there is a permutation of the vertices of $G$ which sends each member of $\mathscr{F}$ into a member of $\mathscr{F}^{\prime}$. It is easy to see that, up to isomorphism, $K_{2}, K_{4}$ and $K_{6}$ have unique one-factorizations. There are six non-isomorphic one-factorizations of $K_{8}$. We shall prove

THEOREM 1. When $n \geqq 4$, there are two non-isomorphic one-factorizations of $K_{2 n}$.

Given any positive integers $i, k$ and $n$, we shall write $d_{i k}$ for the greatest common divisor $(i-k, 2 n-1)$ of $i-k$ and $2 n-1$, and 


$$
v_{i k}=(2 n-1) / d_{i k}
$$

$\equiv$ will denote congruence modulo $2 n-1$.

\section{Divisions}

Suppose $F_{i_{1}}, F_{i_{2}}, \cdots, F_{i t}$ are members of a factorization $\mathscr{F}$ of a graph $G$. We say that they form a t-division if $F_{i_{1}} \cup F_{i_{2}} \cup \cdots \cup F_{i_{t}}$ is a disconnected graph, and refer to the vertex-sets of the components of the union as the components of the division. If $F_{i_{1}}, F_{i_{2}}, \cdots, F_{i_{\mathrm{t}}}$ are a $t$-division then $F_{i}, F_{i_{\beta}}$ will necessarily be a 2 division if $\alpha \neq \beta$, and each component of the $t$-division will be a union of the components of the 2-division.

If $\mathscr{F}$ is a one-factorization of $G$ then $F_{i_{1}} \cup F_{i_{2}} \cup \cdots \cup F_{i_{t}}$ is regular of degree $t$. Therefore each component of a $t$-division contains more than $t$ vertices. In particular if $G$ is of order $2 n$ then an $(n-1)$-division has two components of order $n$; no $n$-division can occur. (In fact no $(n-1)$-division can occur when $n$ is odd, as the components have one-factors and consequently must be of even order.)

LEMMA 1. If $G_{i}$ and $G_{k}$ are any two factors in $\mathscr{G}_{2 n}$ then $G_{i} \cup G_{k}$ consists of a cycle of length $v_{i k}+1$ and $\frac{1}{2}\left(d_{i k}-1\right)$ cycles of length $2 v_{i k}$.

ProOF. Since $G_{i} \cup G_{k}$ is a regular graph of degree 2, it is a union of disjoint cycles. If one such cycle is

$$
\gamma_{0}, \gamma_{1}, \cdots, \gamma_{t},
$$

where $\gamma_{0}=\gamma_{t}$, it is necessarily true that $\left\{\gamma_{0}, \gamma_{1}\right\},\left\{\gamma_{2}, \gamma_{3}\right\}, \cdots,\left\{\gamma_{2 x}, \gamma_{2 x+1}\right\}, \cdots$ are all in the same one-factor. The edge $\left\{\gamma_{t-1}, \gamma_{0}\right\}$ cannot be in this one-factor, because $\gamma_{0}$ cannot have degree 2 in a one-factor. So all the cycles are of even length, and the edges are alternately in $G_{i}$ and $G_{k}$.

Suppose the cycle containing vertex $2 n$ is of length $2 m$; write it as

$$
\alpha_{0}, \alpha_{1}, \cdots, \alpha_{2 m-1}, \alpha_{2 m}
$$

where $\alpha_{0}=\alpha_{2 m}=2 n$. Without loss of generality we can assume $\alpha_{1}=i$ and $\alpha_{2 m-1}=k$. Since (3) is a cycle, $\alpha_{2 x-1} \neq k$ when $0<x<m$. The edge $\left\{\alpha_{2 x}, \alpha_{2 x+1}\right\}$ belongs to $G_{i}$, and from (1) the typical edge of $G_{i}$ (other than $\{2 n, i\}$ ) has form $\{j, 2 i-j\}$, so

$$
\alpha_{2 x+1} \equiv 2 i-\alpha_{2 x}
$$

and similarly

$$
\alpha_{2 x} \equiv 2 k-\alpha_{2 x-1} \text {, }
$$

provided $\alpha_{2 x}$ is not $2 n$ and $\alpha_{2 x-1}$ is not $i$ or $k$. So

$$
\alpha_{2 x+1} \equiv 2(i-k)+\alpha_{2 x-1}
$$




$$
\equiv 2 x(i-k)+i
$$

provided $1 \leqq x \leqq m-1$. In particular

$$
\alpha_{2 x+1}=k \text { if and only if }(2 x+1)(i-k) \equiv 0,
$$

provided that $\alpha_{t} \neq i, k$ or $2 n$ for $1<t<2 x+1$. Since $x=m-1$ is to be the smallest positive solution of $\alpha_{2 x+1}=k$, and $2 x+1=v_{i k}$ is the smallest positive solution of $(2 x+1)(i-k) \equiv 0$, we have $2 m=v_{i k}+1$, and the cycle (3) is of length $v_{i k}+1$.

Now consider any $z$ not in the cycle (3). Suppose that the cycle containing $z$ in $G_{i} \cup G_{k}$ if of length $2 l$; call it

$$
\beta_{0}, \beta_{1}, \cdots, \beta_{2 l}
$$

where $z=\beta_{0}=\beta_{2 l}$. Without loss of generality we may assume $\left\{\beta_{0}, \beta_{1}\right\} \in G_{i}$ and $\left\{\beta_{2 l-1}, \beta_{2 l}\right\} \in G_{k}$. Analogously to (4) and (5) we obtain

and consequently

$$
\begin{aligned}
& \beta_{2 x+1} \equiv 2 i-\beta_{2 x}, \\
& \beta_{2 x} \equiv 2 k-\beta_{2 x-1},
\end{aligned}
$$

$$
\beta_{2 x+1} \equiv 2(x-y)(i-k)+\beta_{2 y+1} .
$$

Since none of $i, k$ or $2 n$ can occur in this cycle, we need place no restriction on this equation, provided the subscripts $2 x+1$ and $2 y+1$ are reduced modulo $2 k$, so

$$
\beta_{2 x+1}=\beta_{2 y+1} \text { if and only if } 2(x-y)(i-k) \equiv 0 .
$$

By definition $\beta_{2 x+1}=\beta_{2 y+1}$ if and only if $2 l$ divides $(2 x+1)-(2 y+1)$, that is, if and only if $l$ divides $x-y$. But $2(x-y)(i-k) \equiv 0$ if and only if $v_{i k}$ divides $2(x-y)$, that is, if and only if $v_{i k}$ divides $x-y$ (since $v_{i k}$ is odd). So $l=v_{i k}$, and the cycle (8) has length $2 v_{i k}$.

We have shown that $G_{i} \cup G_{k}$ has one cycle of length $v_{i k}+1$ and all other cycles of length $2 v_{i k}$. Since $G$ has $2 n$ vertices, the number of cycles of length $2 v_{i k}$ must be

that is $\frac{1}{2}\left(d_{i k}-1\right)$.

$$
\frac{2 n-v_{i k}-1}{2 v_{i k}}
$$

THEOREM 2. When $n>2, \mathscr{G}_{2 n}$ cannot contain an $(n-1)$-division.

Proof. An ( $n-1)$-division would have two components of order $n$. Suppose $n>2$, so that $n-1 \geqq 2$, and let $G_{i}$ and $G_{k}$ be two different factors in an $(n-1)$ division. The 2-division $\left\{G_{i}, G_{k}\right\}$ has one component of size $v_{i k}+1$ and $\left(d_{i j}-1\right)$ components of size $2 v_{i k}$. So one of the components of the $(n-1)$-division must be a union of disjoint sets of size $2 v_{t k}$. So $v_{i k}$ divides $n$; since $v_{i k}$ also divides $2 n-1$ 
we have $v_{i k}=1$ and $d_{i k}=2 n-1$, which is impossible since $1 \leqq i, k \leqq 2 n-1$ and $i \neq k$.

THEOREM 3. If $n \neq 5$, no 2-division of $\mathscr{G}_{2 n}$ has a component of order $2 n-4$.

Proof. Consider the 2-division $\left\{G_{i}, G_{k}\right\}$ whose components have sizes $2 v_{i k}$ and $v_{i k}+1$. Since $v_{i k}$ divides the odd number $2 n-1$ and as observed in the above proof $v_{i k}>1, v_{i k} \geqq 3$. If $v_{i k}+1=2 n-4$ we have $v_{i k}=2 n-5\left|2 n-1, v_{i k}\right| 4$, which is a contradiction. If $2 v_{i k}=2 n-4$ then $v_{i k} \mid(2 n-4,2 n-1)$, so $v_{i k} \mid 3$; so $v_{i k}=3$ and $n=5$.

\section{Proof of theorem 1}

We shall exhibit:

(A) a one-factorization $\mathscr{H}_{2 n}$ of $K_{2 n}$ which contains an $(n-1)$-division, for every even $n$;

(B) a one-factorization $\mathscr{L}_{2 n}$ of $K_{2 n}$ which contains a 2-division with a component of order $2 n-4$, for every odd $n$ greater than 5 ;

(C) two non-isomorphic one-factorizations of $K_{10}$.

Theorem 2 together with (A) proves Theorem 1 for even $n$, Theorem 3 together with (B) proves Theorem 1 for odd $n$ greater than 5 , and (C) completes the proof.

PART (A). In this case $n$ is even, so $K_{n}$ is one-factorable. Label the vertices of $K_{2 n}$ as $1_{1}, 2_{1}, \cdots, n_{1}, 1_{2}, 2_{2}, \cdots, n_{2}$, and let $F_{\alpha, 1}, F_{\alpha, 2}, \cdots, F_{\alpha, n-1}$ be the factors in some one-factorization of the $K_{n}$ with vertices $1_{\alpha}, 2_{\alpha}, \cdots, n_{\alpha}$.

Then write

$$
\begin{array}{ll}
H_{i}=F_{1 . i} \cup F_{2 . i} & i=1,2, \cdots, n-1 \\
H_{i}=X_{i-n+1} & i=n, n+1, \cdots, 2 n-1
\end{array}
$$

where $X_{i}$ are as defined in (2). Write $\mathscr{H}_{2 n}=\left\{H_{1}, H_{2}, \cdots, H_{2 n-1}\right\}$. Then clearly $\mathscr{H}_{2 n}$ is a one-factorization of $K_{2 n}$ and contains an $(n-1)$-division

$$
\left\{H_{1}, H_{2}, \cdots, H_{n-1}\right\} \text {. }
$$

PART (B). When $n$ is odd, write $n=2 m+1$, and write the vertices of $K_{4 m+2}$ as $1_{1}, 2_{1}, \cdots,(2 m+1)_{1}, 1_{2}, 2_{2}, \cdots,(2 m+1)_{2}$. Write $G_{\alpha, 1}, G_{\alpha .2}, \cdots, G_{\alpha .2 m}$ for the factors in the one-factorization $\mathscr{G}_{2 m+2}$ of the $K_{m+2}$ with vertices $1_{\alpha}, 2_{\alpha}, \cdots,(2 m+2)_{\alpha}$, as defined in (1), for $\alpha=1,2$; write $G_{\alpha, i}^{*}$ for $G_{\alpha, i}$ with $\left(i_{\alpha}\right.$, $\left.(2 m+2)_{\alpha}\right)$ deleted; and write

$$
L_{i}^{*}=G_{1, i}^{*} \cup G_{2, i}^{*} \cup\left\{\left(i_{1}, i_{2}\right)\right\} .
$$

Now carry out the vertex-permutation defined by

$$
\begin{aligned}
(2 m+2-i)_{\alpha} & \mapsto(2 i)_{\alpha} \\
(i+1)_{\alpha} & \mapsto(2 i+1)_{\alpha} \\
1_{\alpha} & \leftrightarrow 1_{\alpha}
\end{aligned}
$$


for $i=1,2, \cdots, m$ and $a=1,2$, writing $L_{i}$ for the result of applying the permutation to $L_{i}^{*}$. Then $L_{1}, L_{2}, \cdots, L_{2 m+1}$ are edge-disjoint one-factors of $K_{4 m+2}$, and their union contains all the edges of the form $\left(j_{1}, k_{1}\right)$ and $\left(j_{2}, k_{2}\right)$ where $j \neq k$ and all the edges $\left(j_{1}, j_{2}\right)$, but no edge of the form $\left(j_{1}, k_{2}\right)$ with $j \neq k$. Now define

$$
L_{i}=X_{i-2 m}, i=2 m+2,2 m+3, \cdots, 4 m+1
$$

where $X_{i}$ are as defined in (2) with $n$ replaced by $2 m+1$.

$$
\begin{aligned}
& \mathscr{L}_{4 m+2}=\left\{L_{1}, L_{2}, \cdots, L_{4 m+1}\right\} \text { is a one-factorization of } K_{4 m+2} . \text { Now } \\
& L_{1}=\left\{\left(1_{1}, 1_{2}\right),\left(2_{1}, 3_{1}\right), \cdots,\left((2 x)_{1},(2 x+1)_{1}\right), \cdots,\left((2 m)_{1},(2 m+1)_{1}\right),\right. \\
&\left.\left(2_{2}, 3_{2}\right), \cdots,\left((2 x)_{2},(2 x+1)_{2}\right), \cdots,\left((2 m)_{2},(2 m+1)_{2}\right)\right\}, \\
& L_{2 m+4}=\left\{\left(1_{1},(2 m-1)_{2}\right),\left(2_{1},(2 m)_{2}\right),\left(3_{1},(2 m+1)_{2}\right),\left(4_{1}, 1_{2}\right), \cdots,\left((2 m+1)_{1},\right.\right. \\
&\left.\left.(2 m-2)_{2}\right)\right\},
\end{aligned}
$$

and $L_{1} \cup L_{2 m+4}$ contains the cycle

$$
1_{1}, 1_{2}, 4_{1}, 5_{1}, 2_{2}, 3_{2}, 6_{1}, 7_{1}, \cdots,(2 m-1)_{2}, 1_{1}
$$

of length $4 m-2$, that is $2 n-4$.

PART (C). Suitable 1-factorizations of $K_{10}$ are $G_{10}$, which contains the 3-division $\left\{F_{1}, F_{4}, F_{7}\right\}$, and

$\begin{array}{lllll}\{(1,10), & (2,3), & (4,5), & (6,7), & (8,9)\}, \\ \{(2,10), & (1,4), & (3,9), & (5,6), & (7,8)\}, \\ \{(3,10), & (1,8), & (2,4), & (5,7), & (6,9)\}, \\ \{(4,10), & (1,3), & (2,6), & (5,8), & (7,9)\}, \\ \{(5,10), & (1,9), & (2,7), & (3,8), & (4,6)\}, \\ \{(6,10), & (1,5), & (2,9), & (3,7), & (4,8)\}, \\ \{(7,10), & (1,2), & (3,4), & (5,9), & (6,8)\}, \\ \{(8,10), & (1,7), & (2,5), & (3,6), & (4,9)\}, \\ \{(9,10), & (1,6), & (2,8), & (3,5), & (4,7)\},\end{array}$

which contains no 3-division.

\section{Reference}

[1] F. Harary, Graph Theory, (Addison-Wesley, Reading, Mass., 1969).

University of Newcastle

New South Wales

Australia 